\title{
Branching ratios of $x$-ray photons from dielectronic recombination processes in H-like titanium ions
}

\author{
B. E. O'Rourke* and F. J. Currell \\ Department of Physics, Queens University Belfast, Belfast BT7 1NN, United Kingdom \\ H. Kuramoto, S. Ohtani, and H. Watanabe \\ University of Electro-Communications, Chofu, Tokyo 182-8585, Japan \\ Y. M. Li \\ Institute of Applied Physics and Computational Mathematics, Beijing 100088, China \\ T. Tawara \\ Max Planck Institute für Kernphysik, D-69117 Heidelberg, Germany
}

X. M. Tong

Institute of Materials Science and Center for Computational Sciences, University of Tsukuba, Tsukuba, Ibaraki 305-8573, Japan

(Received 31 March 2008; published 16 June 2008)

\begin{abstract}
In dielectronic recombination of hydrogenlike ions an intermediate doubly excited heliumlike ion is formed. Since the $K$ shell is empty, both excited electrons can decay sequentially to the ground state. In this paper we analyze the $\mathrm{x}$-ray radiation emitted from doubly and singly excited heliumlike titanium ions produced inside the Tokyo electron beam ion trap. Theoretical population densities of the singly excited states after the first transition and the transition probabilities of these states into the ground state were also calculated. This allowed theoretical branching ratios to be determined for each manifold. These branching ratios are compared to the experimentally obtained $\mathrm{x}$-ray distribution by fitting across the relevant peak using a convolution of the theoretically obtained resonance strengths and energies. By taking into account $2 E 1$ transitions which are not observed in the experiment, the measured and calculated ratios agree well. This method provides a valuable insight into the transition dynamics of excited highly charged ions.
\end{abstract}

DOI: 10.1103/PhysRevA.77.062709

PACS number(s): 34.80.Lx, 32.80.Hd, 32.30.Rj

\section{INTRODUCTION}

Dielectronic recombination (DR) is a two-step electronion recombination process [1], involving the resonant capture of an electron with accompanying excitation of a bound electron followed by radiative stabilization. DR is known to play a key role in high-temperature laboratory and astrophysical plasmas. Knowledge of DR cross sections is therefore essential for accurate modeling and characterization of astrophysical plasmas $[2,3]$. Through this process, the charge distributions in plasmas and thus their physical and radiative properties drastically change, resulting in significant loss of energy from the plasma through intense $\mathrm{x}$-ray radiation. This is important in hot fusion plasmas [4], where titanium can be present as an impurity ion.

For $\Delta n>0$ resonances, individual resonances may be grouped together into manifolds normally labeled using an inverse auger notation, e.g., KLn. In this notation a bound $K$-shell electron is excited and a continuum electron is captured, with one of the electrons ending up in the $L$ shell and the other in the $n$ shell.

It is instructive to first consider $K L L$ in a He-like ion, $\mathrm{A}^{(z-2)+}\left(1 s^{2}\right)$, as an example. The following process is expected to occur:

\footnotetext{
*b.orourke@qub.ac.uk
}

$$
\begin{gathered}
e+A^{(z-2)+}\left(1 s^{2}\right) \rightarrow A^{(z-3)+}\left(1 s 2 l 2 l^{\prime}\right) \\
\downarrow \\
A^{(z-3)+}\left(1 s^{2} 2 l^{\prime}\right)+h \nu_{1},
\end{gathered}
$$

where $l$ represents the angular momentum quantum number. One of the $K$-shell electrons in the ion is excited into the $2 l$ (or $2 l^{\prime}$ ) state, while the incident electron, losing its energy after exciting the $K$-shell electron, is captured into the $2 l^{\prime}$ (or $2 l$ ) state, thus forming the doubly excited state $1 s 2 l 2 l^{\prime}$. As there is one single $K$-shell vacancy in the ion, only one of the two electrons in the doubly excited $2 l 2 l^{\prime}$ state decays into the $K$ shell, emitting a $K \alpha \mathrm{x}$ ray $\left(h \nu_{1}\right.$ with its energy of $E_{h \nu_{1}}$, this is often called the $K \alpha$ satellite), and, finally, a Li-like ion $\left(1 s^{2} 2 l\right)$ is formed and stabilized. This process is shown schematically in Fig. 1(a).

On the other hand, in KLL DR of a H-like ion, the process looks similar to that shown in Eq. (1) but two steps are involved:

$$
\begin{gathered}
e+A^{(z-1)+}(1 s) \rightarrow A^{(z-2)+}\left(2 l 2 l^{\prime}\right) \\
\downarrow \\
A^{(z-2)+}\left(1 s 2 l^{\prime}\right)+h \nu_{1}
\end{gathered}
$$


(b)
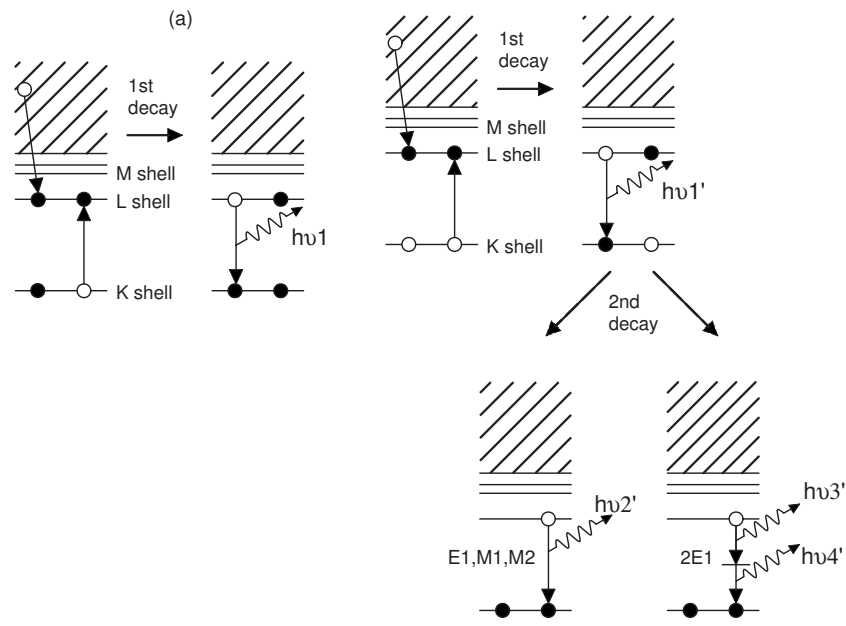

FIG. 1. Schematic representation of the $K L L$ DR process into (a) a He-like ion and (b) a H-like ion and the resulting possibilities for x-ray emission. A $K L L$ DR resonance creates a doubly excited state with both electrons in the $n=2$ state (in this particular example). In DR into He-like ions, only a single $\mathrm{x}$ ray $\left(h \nu_{1}\right)$ is emitted. In contrast, in DR into H-like ions, the doubly excited state $\left(2 l 2 l^{\prime}\right)$ decays emitting the first $\mathrm{x}$ ray $\left(h \nu_{1}^{\prime}\right)$ into the singly excited state $(1 s 2 l)$, which eventually decays to the $1 s^{2}$ ground state, emitting the second $\mathrm{x}$ ray $\left(h \nu_{2}^{\prime}\right.$ or $h \nu_{3}^{\prime}$ and $h \nu_{4}^{\prime}$ combined), whose feature depends on the excited state formed. $E_{h \nu_{1}^{\prime}}>E_{h \nu_{1}}, E_{h \nu_{2}^{\prime}}=E_{h \nu_{1}}$, and also $E_{h \nu_{3}^{\prime}}$ $+E_{h \nu_{4}^{\prime}}=E_{h \nu_{2}^{\prime}}$. Note that $E 1, M 1, M 2$, and $2 E 1$ denote the decay modes shown in Table III.

$$
A^{(z-2)+}\left(1 s^{2}\right)+h \nu_{2} \text {. }
$$

There is a very important and distinctive difference; there are two $K$-shell vacancies available [see Fig. 1(b)]. Thus, two transitions can occur from this doubly excited state; first, one of the two electrons decays into the $K$ shell, emitting the first $K \alpha \mathrm{x}$ ray $\left(h \nu_{1}^{\prime}\right)$, whose energy $\left(E_{h \nu_{1}^{\prime}}\right)$ is slightly higher than that $\left(E_{h \nu_{1}}\right)$ in DR of the isonuclear He-like ion $\left[h \nu_{1}\right.$ in Fig. $1(\mathrm{a})]$ as there is no screening $1 \mathrm{~s}$ electron. This is often called the hypersatellite, $K \alpha^{h}$. A singly excited state $(1 s 2 l)$ is formed which subsequently decays to the ground state $\left(1 s^{2}\right)$, mostly through a dipole or quadrupole transition resulting in emission of the second $\mathrm{x}$ ray $\left(h \nu_{2}^{\prime}\right)$. The energy $\left(E_{h \nu_{2}^{\prime}}\right)$ of the second $\mathrm{x}$ ray is practically the same as that $\left(E_{h \nu_{1}}\right)$ emitted by the isonuclear He-like ion.

Some of the singly excited states formed after emission of the first photon can decay only through a two-photon $(2 E 1)$ transition. In this case, the energy sharing results in emission of pairs of photons, with a symmetric energy distribution. The summed energy of two photons is equal to the total transition energy $\left(E_{h \nu_{3}^{\prime}}+E_{h \nu_{4}^{\prime}}=E_{h \nu_{2}^{\prime}}\right)$. These decay schemes are also shown in Fig. 1(b). It should be noted that the energy difference between $\left(E_{h \nu_{1}^{\prime}}\right)$ and $\left(E_{h \nu_{2}^{\prime}}\right)$ strongly depends on the atomic number of the ions considered. Furthermore, the situation in $K L M$ DR resonance forming the doubly excited $\left(2 l 3 l^{\prime}\right)$ states is much more complicated since there are two possible main decay channels from the initial doubly excited state into the ground state via the intermediate, singly excited state, including some cascade channels, thus emitting two $\mathrm{x}$ rays with slightly different energies in a single DR process.

Ion storage rings have been used extensively to measure $\Delta n=0$ resonances in heavy few-electron ions $[5,6]$, or $\Delta n$ $>0$ processes in lighter ions [7] by detuning the electron cooler for short periods. Recently, KLL DR in H-like Xe [8] and $\mathrm{U}$ [9] have been measured by employing stochastic cooling, freeing up the electron cooler to be used as an electron target over a wider energy range. The use of gas targets to measure cross sections for radiative transfer and excitation (RTE) has also been reported, the RTE process being essentially equivalent to DR but with bound rather than free electrons $[10,11]$.

Electron beam ion traps and sources (EBITs and EBISs) have also been used to probe $\Delta n>0$ resonances in heavy few-electron ions. DR cross sections have been obtained by modeling the charge balance of the plasma in the trap either through estimations based on the ratios of extracted ions or emitted x-ray distributions. Ali et al. [12,13] used extracted ions from the Kansas EBIS to measure DR cross sections in He-like argon. Similarly DeWitt et al. used the Livermore EBIT to measure resonance strengths in H-like argon [14] and Ne-like [15] and F-like [16] xenon. Recently a variation of this extracted ion method was used to determine the $K L L$ and $K L M$ DR resonance strengths of $\mathrm{C}$ - to He-like iodine ions [17] and Be- to He-like Fe, Y, Ho, and $\mathrm{Bi}$ ions [18]. These experiments are performed by measuring the ratio of extracted ions for two neighboring charge species as a function of beam energy. DR cross sections are then determined by normalization to theoretical ionization cross sections with charge exchange and escape corrected for by subtraction of a slowly varying function.

DR cross sections can also be measured without extracting the ions by measuring the $\mathrm{x}$ rays emitted from the excited highly charged ions as they decay in the trap while the electron beam is scanned through the resonance. In this case $90^{\circ}$ differential resonance strengths $d S^{K L n} / d \Omega$ of a manifold $K L n$ are usually obtained by normalization to theoretical radiative recombination (RR) cross sections using the formula

$$
\frac{d S^{K L n}}{d \Omega}=\frac{I^{K L n}}{I^{\mathrm{RR}}}\left(\frac{d \sigma^{\mathrm{RR}}}{d \Omega} \Delta E\right),
$$

where $d \sigma^{\mathrm{RR}} / d \Omega$ and $I^{\mathrm{RR}}$ are the differential RR cross sections and measured intensities of RR photons into the same sublevel, respectively. $I^{K L n}$ is the intensity of photons from the $K L n$ resonance summed over an energy interval $\Delta E$. This method has the advantage that normalization is performed with respect to theoretical RR cross sections which are in general more accurately determined than those for electron impact ionization. This method also allows for information on the branching ratios of the decay of the doubly excited intermediate states to be extracted.

On the other hand the trap region of an EBIT usually contains a broad distribution of ions each of which contributes to the total $\mathrm{x}$-ray spectra observed. In order to determine resonance strengths for individual charge states, this charge state balance needs to be determined by a complicated fitting 
procedure. One common solution is to try to push the charge state balance toward as pure a distribution as possible. This can be achieved by fixing the beam energy just below the He-like ionization threshold, which produces a predominantly He-like ion charge state balance. For this reason previous measurements of DR resonance strengths using the emitted x-ray distribution have focused on He-like ions, including measurements at the LLNL EBIT by Smith et al. on $\mathrm{Ar}^{16+}$ [19] and Knapp et al. on $\mathrm{Ni}^{26+}, \mathrm{Mo}^{40+}, \mathrm{Ba}^{54+}$ [20], and $\mathrm{U}^{90+}[21]$. Previous work by our group at the Tokyo EBIT has also been reported for $\mathrm{Fe}^{24+}[22]$ and $\mathrm{Ti}^{20+}$ [23]. Stateand configuration-resolved resonance strengths for He-like $\operatorname{Ar}$ [24] and Ge [25] were measured at the Heidelberg EBIT, where enhanced resolution was obtained by using very low electron beam currents. Open shell DR in He-, Li- and Belike krypton ions was also studied by Fuchs et al. [26] by fitting the observed resonance peaks with theoretically derived individual charge state components. Recently Chen et al. [27] used a similar method to measure KLL DR cross sections for Be-, B-, and C-like xenon. By crossing the Helike ionization threshold some fraction of $\mathrm{H}$-like ions is also produced and in this way measurements of KLL and $K L M$ DR resonance strengths in $\mathrm{H}$-like titanium via the $\mathrm{x}$-ray method [28] was reported. Since $\mathrm{x}$ rays are typically observed at $90^{\circ}$ to the electron beam direction, the polarization of photons from individual transitions must be calculated and folded into the fitting procedure.

In the high- $Z$ region resonance strengths [29] and energies [30] of KLL DR resonances were also measured for B- to He-like mercury ions $\left(\mathrm{Hg}^{75+}\right.$ to $\left.\mathrm{Hg}^{78+}\right)$ at the Heidelberg EBIT via the emitted $\mathrm{x}$-ray spectra. State resolved resonance energies were measured with an absolute accuracy of $\pm 14 \mathrm{eV}$ and compared to theoretical calculations [31]. Measurements at this level of accuracy provide a test of quantum electrodynamics (QED) effects in few electrons ions. In this case good agreement between experiment and theory ws obtained for He-like ions although significant discrepancies were found for the other multi-electron systems.

This paper reports a detailed analysis of the x-ray photons observed in our previous experiment on H-like Ti ions [28] with particular emphasis on the branching ratios of the decay of doubly excited states. In the case of H-like DR this can give us an important window on the radiative decay of highly charged He-like ions.

\section{EXPERIMENT}

The present experiment has been performed using the Tokyo EBIT [32] and partial results for the total resonance strengths and branching ratios were reported by Watanabe et al. [28]. The electron beam current during the present measurements was kept constant at about $50 \mathrm{~mA}$, confined within the estimated radius of about $30 \mu \mathrm{m}$ (the corresponding electron current density is about $\left.1800 \mathrm{~A} / \mathrm{cm}^{2}\right)$. Lowcharged $\mathrm{Ti}$ ions were periodically injected into the trap from a metal vapor vacuum arc (MEVVA) ion source. To look for the DR resonance, the electron energy was varied over the range of 3-11 keV using a triangular wave form with a period of $4 \mathrm{~ms}$ and thus a scan speed of $4 \mathrm{~V} / \mu \mathrm{s}$. Under such
TABLE I. Calculated partial resonance strength $\left(S_{p}\right)$, "primary" X-ray energy $(\mathrm{keV})$, polarization $(P)$, and differential strength $(d S / d \Omega)$ for the 14 strongest $K L L$ resonance lines formed via DR into H-like Ti ions. Partial and differential (at 90 ) resonance strengths are given in units of $10^{-20} \mathrm{~cm}^{2} \mathrm{eV}$ and $10^{-20} \mathrm{~cm}^{2} \mathrm{eV} / \mathrm{sr}$, respectively. The integrated partial and differential strengths (total strength) are also given.

\begin{tabular}{lcccc}
\hline \hline Transition & $E_{h \nu}$ & $S_{p}$ & $P[35]$ & $d S / d \Omega$ \\
\hline $2 s^{2}{ }^{1} S_{0} \rightarrow 1 s 2 p{ }^{1} P_{1}$ & 4.888 & 1.28 & 0 & 0.102 \\
$2 s^{2}{ }^{1} S_{0} \rightarrow 1 s 2 p{ }^{3} P_{1}$ & 4.911 & 0.40 & 0 & 0.031 \\
$2 s 2 p{ }^{1} P_{1} \rightarrow 1 s 2 s{ }^{1} S_{0}$ & 4.958 & 8.56 & 0.999 & 1.021 \\
$2 s 2 p{ }^{1} P_{1} \rightarrow 1 s 2 s{ }^{3} S_{1}$ & 4.982 & 0.14 & -0.997 & 0.009 \\
$2 s 2 p{ }^{3} P_{2} \rightarrow 1 s 2 s{ }^{3} S_{1}$ & 4.950 & 2.37 & 0.447 & 0.222 \\
$2 s 2 p{ }^{3} P_{1} \rightarrow 1 s 2 s{ }^{3} S_{1}$ & 4.944 & 1.65 & 0.150 & 0.138 \\
$2 s 2 p{ }^{3} P_{0} \rightarrow 1 s 2 s{ }^{3} S_{1}$ & 4.940 & 0.48 & 0 & 0.038 \\
$2 p^{2}{ }^{1} S_{0} \rightarrow 1 s 2 p{ }^{1} P_{1}$ & 4.972 & 0.91 & 0 & 0.072 \\
$2 p^{2}{ }^{1} D_{2} \rightarrow 1 s 2 p{ }^{1} P_{1}$ & 4.938 & 22.14 & 0.600 & 2.202 \\
$2 p^{2}{ }^{1} D_{2} \rightarrow 1 s 2 p{ }^{3} P_{2}$ & 4.956 & 3.36 & -1.000 & 0.201 \\
$2 p^{2}{ }^{3} P_{2} \rightarrow 1 s 2 p{ }^{1} P_{1}$ & 4.926 & 0.91 & 0.600 & 0.091 \\
$2 p^{2}{ }^{3} P_{2} \rightarrow 1 s 2 p{ }^{3} P_{1}$ & 4.950 & 2.46 & 0.600 & 0.245 \\
$2 p^{2}{ }^{3} P_{2} \rightarrow 1 s 2 p{ }^{3} P_{2}$ & 4.942 & 5.52 & -1.000 & 0.329 \\
$2 p^{2}{ }^{3} P_{0} \rightarrow 1 s 2 p{ }^{3} P_{1}$ & 4.940 & 0.08 & 0 & 0.006 \\
& $\Sigma S_{p}=50.26$ & & $\Sigma d S / d \Omega=4.708$ \\
\hline \hline
\end{tabular}

conditions, about $0.5 \%$ of the primary ions confined in the trap region are estimated to be lost due to DR collisions when passing through the strongest resonance. In order to avoid significant accumulation of impurity heavy ions, all the trapped ions were dumped every $1 \mathrm{~s}$.

Throughout this $1 \mathrm{~s}$ period, $\mathrm{x}$ rays were observed with a pure $\mathrm{Ge}$ detector placed at $90^{\circ}$ with the respect to the electron beam direction. For each detected $\mathrm{x}$-ray event, the $\mathrm{x}$-ray energy, electron beam energy, and time since injection were logged using a multiparameter system [33] in list mode.

\section{THEORY}

The resonance energies and strengths of DR of H-like Ti ions were calculated based upon a model proposed by LaGattuta and Hahn [34]. Table I lists the 14 strongest $K L L$ DR resonances together with their calculated $\mathrm{x}$-ray energies, polarizations of the emitted $\mathrm{x}$ rays, partial resonance strengths and differential strengths at $90^{\circ}$. It is found from the present calculations that the transitions listed in Table I make up more than $99 \%$ of the total (theoretical) resonance strength. In each case, the differential resonance strength of a particular line at $90^{\circ}$ to the direction of propagation of the electron beam, where the present observation was performed, was estimated from the calculated partial strength $S_{p}$, assuming that all the x-ray emissions are of the electric dipole type, through the following formula:

$$
d S / d \Omega_{90^{\circ}}=3 S_{p} /[4 \pi(3-P)] .
$$

Here $P$ represents the polarization factor of the emitted $\mathrm{x}$ ray, which can be calculated using a relativistic multiconfiguration Dirac-Fock model [35]. 
TABLE II. Populations of singly excited He-like ions after $K L L$ DR into H-like Ti. The type of decay from each excited state to the ground state is also shown. Transition rates for each decay are given in units of $\mathrm{s}^{-1}$ with numbers in parentheses signifying powers of 10. Differential resonance strengths are in units of $10^{-20} \mathrm{~cm}^{2} \mathrm{eV} / \mathrm{sr}$. BR indicates the branching ratio.

\begin{tabular}{|c|c|c|c|c|c|c|}
\hline Initial & $\%$ & Final & Type & $E_{h \nu}$ & Rate $\left(\mathrm{s}^{-1}\right)$ & BR \\
\hline \multirow[t]{3}{*}{$1 s 2 s{ }^{1} S_{0}$} & \multirow[t]{3}{*}{17.0} & $1 s^{2}{ }^{1} S_{0}$ & $2 E 1$ & & $1.47(9)^{\mathrm{a}}$ & 1 \\
\hline & & $1 s 2 s^{3} S_{1}$ & $M 1$ & 0.026 & $2.98(1)^{\mathrm{b}}$ & 0 \\
\hline & & $1 s 2 s^{3} P_{1}$ & $E 1$ & 0.001 & $2.98(3)^{\mathrm{b}}$ & 0 \\
\hline $1 s 2 s^{3} S_{1}$ & 9.2 & $1 s^{2}{ }^{1} S_{0}$ & $M 1$ & 4.702 & $3.88(7)^{\mathrm{c}}$ & 1 \\
\hline \multirow[t]{3}{*}{$1 s 2 p{ }^{1} P_{1}$} & \multirow[t]{3}{*}{50.1} & $1 s^{2}{ }^{1} S_{0}$ & $E 1$ & 4.749 & $2.401(14)^{\mathrm{d}}$ & 1 \\
\hline & & $1 s 2 s^{3} S_{1}$ & $E 1$ & 0.022 & $1.02(8)^{\mathrm{b}}$ & 0 \\
\hline & & $1 s 2 s{ }^{1} S_{0}$ & $E 1$ & 0.048 & $2.28(8)^{\mathrm{b}}$ & 0 \\
\hline \multirow[t]{3}{*}{$1 s 2 p^{3} P_{2}$} & \multirow[t]{3}{*}{17.7} & $1 s^{2}{ }^{1} S_{0}$ & $M 2$ & 4.734 & $1.66(9)^{b}$ & 0.701 \\
\hline & & $1 s 2 s^{3} S_{1}$ & $E 1$ & 0.032 & $7.07(8)^{\mathrm{b}}$ & 0.229 \\
\hline & & $1 s 2 s{ }^{1} S_{0}$ & $M 2$ & 0.006 & $3.57(-5)^{b}$ & 0 \\
\hline \multirow[t]{2}{*}{$1 s 2 p^{3} P_{1}$} & \multirow[t]{2}{*}{5.9} & $1 s^{2}{ }^{1} S_{0}$ & $E 1$ & 4.727 & $1.118(13)^{\mathrm{d}}$ & 1 \\
\hline & & $1 s 2 s^{3} S_{1}$ & $E 1$ & 0.025 & $3.31(8)^{b}$ & 0 \\
\hline $1 s 2 p^{3} P_{0}$ & 0.0 & $1 s 2 s^{3} S_{1}$ & $E 1$ & 0.024 & $2.93(8)^{b}$ & 0 \\
\hline
\end{tabular}

${ }^{\mathrm{a}}$ Drake [36].

${ }^{\mathrm{b}}$ Lin, Johnson and Dalgarno [37].

${ }^{\mathrm{c}}$ Gould, Marrus, and Schmieder [38].

${ }^{\mathrm{d}}$ Drake [39].

By summing the contributions from different initial DR states given in Table I, it is possible to calculate the partial strength of each of the singly excited $1 s 2 l$ substates of Helike $\mathrm{Ti}^{20+}$ formed after the primary $\mathrm{x}$-ray emission in $K L L$ DR into H-like $\mathrm{Ti}^{21+}$ ions. The six possible $1 s 2 l$ substates of the He-like $\mathrm{Ti}^{20+}$ ion and their partial strengths after $K L L$ $\mathrm{DR}$, transition rates, and branching ratios are listed in Table II. From these excited states, the second decay, accompanied by secondary $\mathrm{x}$-ray emission, takes place to the ground state via a $\Delta n=1$ transition or to another $1 s 2 l$ state via a $\Delta n=0$ transition, followed by another $\Delta n=1$ transition to the ground state. From these rates given in Table II, it is possible to determine the branching ratios for decay to the ground state. For all states except for $1 s 2 p^{3} P_{2}$, the branching ratio is found to be practically unity since the transition rate of $\Delta n=0$ decay is several orders of magnitude smaller than that into the ground state. For $1 s 2 p{ }^{3} P^{2}$ state, the decay to the $1 s^{2}$ ground state has a probability of $70.1 \%$, with the other $22.9 \%$ decaying to $1 s 2 s^{3} S^{1}$, which is finally stabilized into the ground state. All the important information described in Tables I and II is summarized diagrammatically in Fig. 2, where the initial $2 l 2 l^{\prime}$ doubly excited states formed via DR, the $1 s 2 l^{\prime}$ singly excited states formed after the first decay, and the ground state are shown. Transitions between the doubly excited states and the singly excited states corresponding to 14 entries in Table I are drawn as solid lines along with the partial resonance strength for each process. The transitions to the ground state involving emission of the second $\mathrm{x}$ ray are drawn as dashed lines. The $1 s 2{ }^{1} S_{0}$ state decays only through a two-photon transition ( $2 E 1)$ which is shown as a dotted line. Although the diagram is not to scale, the ordering of the energy levels has been maintained.

As is clear from Table II and Fig. 2, all the singly excited states formed after the first transition of DR can decay radia- tively to the ground state sufficiently quickly, compared with the scan speed of the electron energy in the present experiment $(4 \mathrm{~V} / \mu \mathrm{s})$ and, thus, all $\mathrm{x}$ rays from their radiative decay should be observed at the same electron beam energy as the primary $\mathrm{x}$ rays of DR. Another important point is that further excitation or reionization back out of the singly excited $(1 s 2 l)$ states formed after the first decay of DR has been estimated to be much slower under the present experimental conditions $\left(10 \mathrm{~ms}\right.$ at $1800 \mathrm{~A} / \mathrm{cm}^{2}$ in a 30 - $\mu \mathrm{m}$-diameter electron beam).

Though the calculated polarization factors for all the doubly excited to the singly excited decays have already been given in Table I [35], it is necessary to know the polarization factors for the singly excited states formed after the first decay when the ratios of the secondary to the primary $\mathrm{x}$-ray intensities are compared with experimental results. To calculate the polarization of the secondary $\mathrm{x}$ rays, the alignment caused by the cascade into the singly excited state needs to be known. The alignments produced in the singly excited states were calculated by considering alignment transfer by cascade transitions [40]. From the calculated alignment the polarization factor $P$ was obtained.

For the electric dipole transition the intensity at $90^{\circ}$ is related to the polarization [see Eq. (4)], whereas for the magnetic dipole transition it is given as [41]

$$
d I / d \Omega_{90^{\circ}}=3 I /[4 \pi(3+P)] .
$$

The angular variation of the emitted x-ray intensities of higher-order multipole transitions cannot be expressed as a simple function of the polarization. It has been shown [42] that the $M 2$ decay in $1 s 2 p^{3} P_{2} \rightarrow 1 s^{2}{ }^{1} S_{0}$ transition of He-like $\mathrm{Fe}$ ions behaves as an electric dipole transition with the same angular distribution given in Eq. (4). It seems reasonable to 


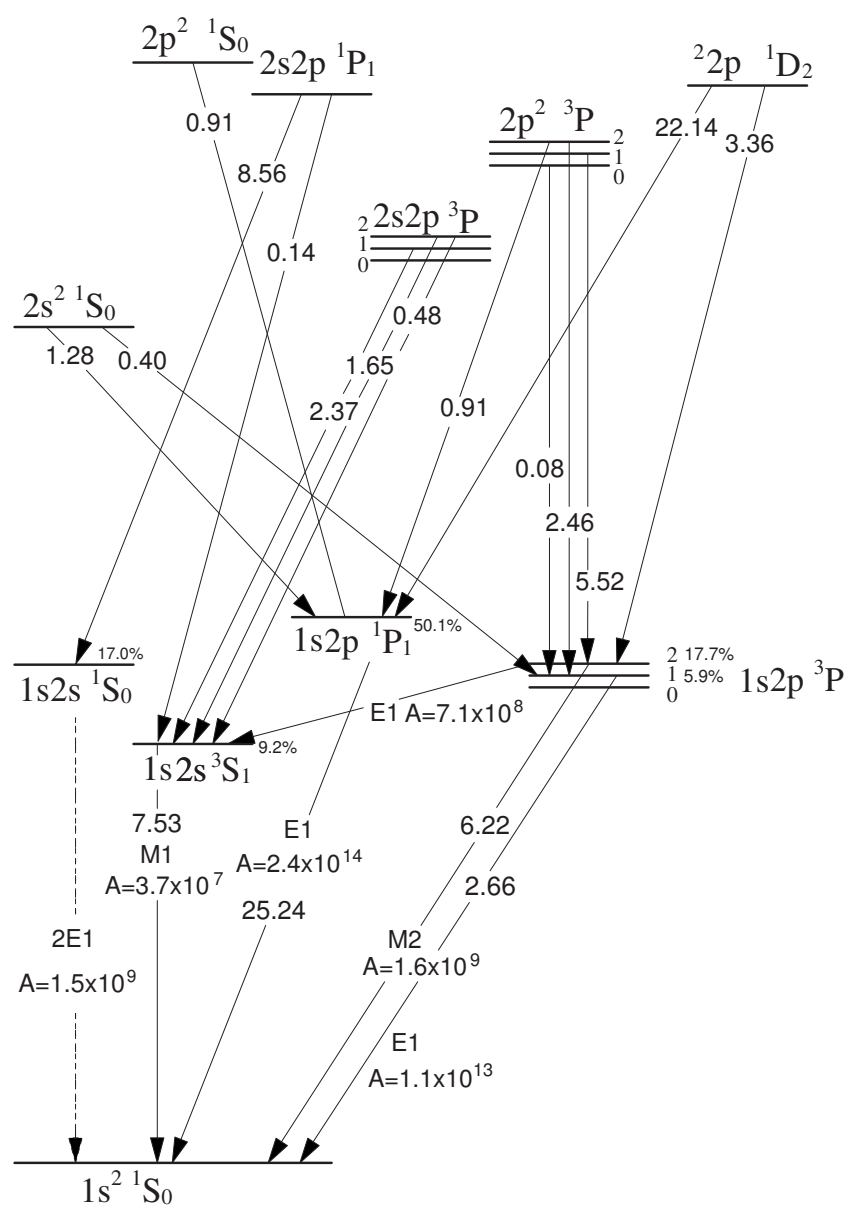

FIG. 2. Grotrian diagram showing the doubly and singly excited states in $\mathrm{He}$-like $\mathrm{Ti}^{20+}$, formed after $K L L \mathrm{DR}$ into $\mathrm{H}$-like $\mathrm{Ti}^{21+}$. The calculated partial resonance strengths associated with each $2 l 2 l^{\prime}$ $\rightarrow 1 s 2 l^{\prime}$ and $1 s 2 l \rightarrow 1 s^{2}$ transition are given in units of $10^{-20} \mathrm{~cm}^{2} \mathrm{eV}$. Note that the total resonance strength of the $K L L$ process is $50.26 \times 10^{-20} \mathrm{~cm}^{2} \mathrm{eV}$ (see Table I). The population of each doubly excited $1 s 2 l$ state after the initial radiative decay and the decay type and calculated transition rates (in $\mathrm{s}^{-1}$ ) for $1 s 2 l$ $\rightarrow 1 s^{2}$ decays to the ground state are also shown. The dotted line shows the $2 E 1$ transition, emitting two $\mathrm{x}$ rays with different energies, which are not detected in the present experiment.

assume here that this is also the case for He-like Ti since the atomic numbers of these two ions are similar.

The calculated polarization and differential resonance strengths at $90^{\circ}$ for each transition are given in Table III. The differential resonance strengths are obtained from the partial resonance strength combined with the correction for the polarization at $90^{\circ}$. Thus, the theoretical ratio of the secondary (Table III) to the primary (Table I) $\mathrm{x}$-ray intensities at $90^{\circ}$, which should be compared with the observed results, is then simply given by

$$
\frac{d S\left(1 s 2 l \rightarrow 1 s^{2}\right) / d \Omega}{d S\left(2 l 2 l^{\prime} \rightarrow 1 s 2 l\right) / d \Omega}=\frac{3.742}{4.708}=0.795 .
$$

It should be noted that the equivalent theoretical ratio can be estimated in a simpler way to be (4.708-1.021)/4.708 $=0.78$ from Table I alone. However, this second estimate ignores the role of the polarization of the emitted $x$ ray. Furthermore, there is an assumption that all the excited states formed in the DR cascade down into the ground state, except for $1 s 2 s{ }^{1} S_{0}$ which decays via $2 E 1$ two-X-ray emissions, neither of which will be fully detected in the appropriate energy interval. Thus, this contribution should be subtracted from the partial DR given in Table I when comparing with the experimental result. This theoretical result is summarized in Table VI (see Sec. IV) where the "expected" fraction of $\mathrm{X}$-ray intensity, normalized to the total intensity for the manifold, is given for each transition group.

The situation in KLM DR of a H-like ion is more complicated. The initial doubly excited states of $K L M$ produced in DR into $\mathrm{H}$-like ion are $2 l 3 l^{\prime}$ states. The possible decays from these states are shown in a simplified form in Fig. 3. The first decay of these states proceeds either to $1 s 3 l$ states, labeled $a$ (the inner channel), or to $1 s 2 l$ states, labeled $b$ (the outer channel) in the figure. Partial resonance strengths for these transitions were calculated in the same way as in the $K L L$ case described above and are also shown in the figure. Many more individual resonances need to be considered in this case in comparison to the $K L L$ transition. It was found that, for the inner-decay channel transitions $(a)$, the strongest 48 transitions contribute $97.9 \%$ of the partial resonance strength, while 90 transitions were needed to cover $99.9 \%$. For the transitions into $1 s 2 l$ states via the outer-decay channel $(b), 30$ individual transitions contribute $99.5 \%$ of partial resonance strength.

By summing the resonance strengths of each individual transition into the different substates the partial resonance strengths were determined for each of the $1 s 3 l$ and $1 s 2 l$ singly excited states. These values are shown in Table IV.

Some of the $1 s 3 p{ }^{1} P_{1}$ and ${ }^{3} P_{1}$ states formed decay directly to the ground $1 s^{2}$ state. These transitions are labeled $c$

TABLE III. Polarizations and effective differential resonance strengths for the $1 s 2 l \rightarrow 1 s^{2}$ transitions. The type of decay from each excited state to the ground state is also shown. Differential resonance strengths are in units of $10^{-20} \mathrm{~cm}^{2} \mathrm{eV} / \mathrm{sr}$.

\begin{tabular}{lccccc}
\hline \hline State & $\mathrm{S}_{p}$ & Decay to $1 s^{2}$ & $E_{h \nu}$ & $P$ & $d S / d \Omega$ \\
\hline $1 s 2 s{ }^{3} S_{1}$ & 7.53 & $M 1$ & 4.702 & -0.11 & 0.620 \\
$1 s 2 p{ }^{1} P_{1}$ & 25.24 & $E 1$ & 4.749 & 0.58 & 2.491 \\
$1 s 2 p{ }^{3} P_{2}$ & 6.22 & $M 2$ & 4.734 & -1 & 0.372 \\
$1 s 2 p{ }^{3} P_{1}$ & 2.66 & $E 1$ & 4.727 & 0.52 & 0.257 \\
& & & & $\Sigma d S / d \Omega=3.742$ \\
\hline
\end{tabular}




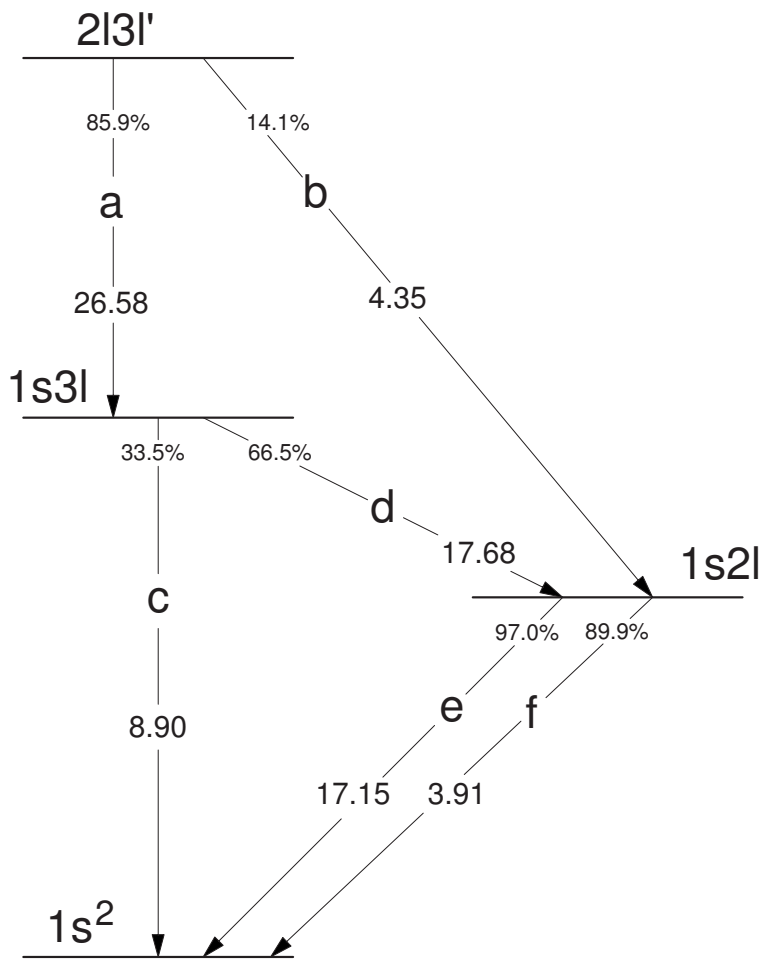

FIG. 3. Grotrian diagram involving transitions from the doubly excited state after $K L M$ DR resonance into $\mathrm{H}$-like Ti ion. Note that there are many different decay channels which form six groups indicated as $a-f$. The transition $a$ represents the inner-decay channel and the transition $b$ the outer-decay channel. The partial strengths for $a$ and $b$ transitions were calculated theoretically as described in text (see Table IV). Partial strengths of $c, e$, and $f$ transitions were determined by taking into account the branching ratios into the individual singly excited levels and subtracting the $2 E 1$ contribution as outlined in the text. Although the lines $e$ and $f$ represent transitions between the same groups of states, they are preceded by different initial transitions, represented by $d$ and $b$, respectively. Therefore they are accounted for separately in the analysis. in Fig. 3. Some of the $1 s 3 l$ states also decay to the $1 s 2 l$ states through transitions labeled $d$ in Fig. 3. The calculated radiative decay rates $\left(A_{r}\right)$ of the main decays from the $1 s 3 p{ }^{1} P_{1}$ and ${ }^{3} P_{1}$ states are also shown in Table V. The partial strengths of these decays were calculated from Table IV and then the branching ratios estimated.

By combining the initial partial strength in Table IV with the calculated branching ratio in Table V, the partial strength of the decay channel $c$ is estimated to be 8.90 $\times 10^{-20} \mathrm{~cm}^{2} \mathrm{eV}$. Then the rest of the $1 s 3 l$ states decay to the $1 s 2 l$ state, with a partial strength of $17.68 \times 10^{-20} \mathrm{~cm}^{2} \mathrm{eV}$.

The partial strength of the decay of $1 s 2 l$ states is slightly complicated due to the cascade contribution from the $1 s 3 \mathrm{l}$ state, in addition to the direct decay channel $b$. To compare the observed results, the contribution of $2 E 1$ decay from the $1 s 2 l$ state to the ground state has to be subtracted from the calculated partial strength, although it is small.

All the information on the calculated partial strengths and branching ratios involving $K L M$ DR of $\mathrm{H}$-like Ti ion is summarized in Fig. 3. Transitions through the channels $a$ and $e$ $+f$ have similar $x$-ray energies since they both involve transitions of the form $2 l \rightarrow 1 s$. These channels cannot be resolved with the present $\mathrm{x}$-ray detector although they again result in significant broadening due to the different screenings provided by the spectator $3 l$ and $1 s$ electrons.

In the same way as $K L L$ the intensity ratios for each transition group in the $K L M$ manifold can be expressed as a fraction of the total intensity. These fractions are given in Table VI. As is clear already from Fig. 3, for the first transition, the inner-decay $\left(2 l 3 l^{\prime} \rightarrow 1 s 3 l^{\prime}\right)$ channel is dominant, compared with the outer-decay $\left(2 l 3 l^{\prime} \rightarrow 1 s 2 l\right)$ channel. This is in agreement with the fact that radiation theory predicts that transitions with $\Delta n=1$ are stronger in comparison with those with $\Delta n=2$ [43]. In the same way we can see that transitions from the $1 s 3 l$ into the $1 s 2 l$ state $(\Delta n$ $=1,66.5 \%)$ are almost twice as likely as direct transitions to the ground $1 s^{2}$ state $(\Delta n=2,33.5 \%)$.

\section{RESULTS}

A portion of the observed x-ray spectrum from $K L L \mathrm{DR}$ into H-like Ti ions is shown in Fig. 4 along with a fit to the

TABLE IV. Partial resonance strengths $\left[S_{p}\left(10^{-20} \mathrm{~cm}^{2} \mathrm{eV}\right)\right]$ into $1 s 3 l$ and $1 s 2 s$ states of the singly excited states formed after the first decay of the KLM $\left(2 l 3 l^{\prime}\right)$ DR resonance state. Relative populations over the manifold are also given.

\begin{tabular}{lccccc}
\hline \hline $1 s 3 l$ & $S_{p}$ & $\%$ & $1 s 2 s$ & $S_{p}$ & $\%$ \\
\hline $1 s 3 s{ }^{1} S_{0}$ & 2.98 & $11.2 \%$ & $1 s 2 s{ }^{1} S_{0}$ & 0.44 & $10.1 \%$ \\
$1 s 3 s{ }^{3} S_{1}$ & 1.27 & $4.8 \%$ & $1 s 2 s{ }^{3} S_{1}$ & 0.82 & $18.9 \%$ \\
$1 s 3 p{ }^{1} P_{1}$ & 8.38 & $31.5 \%$ & $1 s 2 p{ }^{1} P_{1}$ & 2.05 & $47.1 \%$ \\
$1 s 3 p{ }^{3} P_{0}$ & 0.02 & $0.1 \%$ & $1 s 2 p{ }^{3} P_{0}$ & 0.03 & $0.7 \%$ \\
$1 s 3 p{ }^{3} P_{1}$ & 2.27 & $8.5 \%$ & $1 s 2 p{ }^{3} P_{1}$ & 0.64 & $14.7 \%$ \\
$1 s 3 p{ }^{3} P_{2}$ & 2.58 & $9.7 \%$ & $1 s 2 p{ }^{3} P_{2}$ & 0.37 & $8.5 \%$ \\
$1 s 3 d{ }^{1} D_{2}$ & 2.62 & $9.9 \%$ & & & \\
$1 s 3 d{ }^{3} D_{1}$ & 2.23 & $8.4 \%$ & & & \\
$1 s 3 d{ }^{3} D_{2}$ & 2.07 & $7.8 \%$ & & & \\
$1 s 3 p{ }^{3} D_{3}$ & 2.16 & $8.1 \%$ & & & \\
& $\Sigma=26.58$ & & & & \\
\hline
\end{tabular}




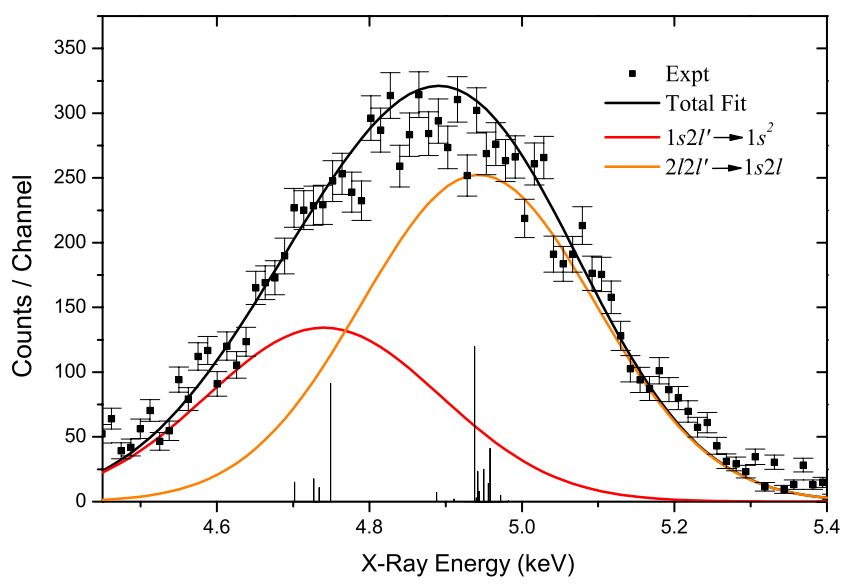

FIG. 4. (Color online) Fit to the observed $K L L$ DR x-ray peak of $\mathrm{H}$-like $\mathrm{Ti}$ ions as a function of x-ray energy. The red (dark gray) line corresponds to the four $1 s 2 l^{\prime} \rightarrow 1 s^{2}$ transition lines (see Table III). The orange (light gray) line is composed of 12 Gaussians including the 14 strongest $2 l 2 l^{\prime} \rightarrow 1 s 2 l$ transition lines (see Table I). The energy and relative intensity of the individual transitions composing the primary and secondary $\mathrm{x}$ rays are indicated with thin vertical bars.

data. The fit consists of 16 individual lines with the Gaussian distributions convoluted with the energy resolution of the detector. 12 of these peaks correspond to the 14 strongest primary transitions given in Table I (there are two lines where a pair of transitions have a difference in x-ray energy of less than $1 \mathrm{eV}$ ) and the other four lines to the secondary transitions outlined in Table II.

The observed $K L L \mathrm{x}$-ray peak is broader than the energy resolution of the detector used $(\approx 350 \mathrm{eV}$, based on the linewidth of the $K L L$ peak for DR into He-like Ti). Transitions in this manifold fall into two groups, one corresponding to the primary transition (see Table I) and the second corresponding to the secondary transition (see Table II). The spacing between the two groups is about $200 \mathrm{eV}$ and is due to the increased screening of the $1 s$ electron compared to that provided by the initial $2 l$ spectator electron.

In the fit, the intensity of each line contributing to the primary and secondary $\mathrm{x}$-ray peaks was fixed according to

TABLE V. Calculated radiative decay rate $\left[A_{r}\left(\mathrm{~s}^{-1}\right)\right.$, figures in parentheses represent powers of 10] and branching ratio (\%) from $1 s 3 p{ }^{1} P_{1}$ and ${ }^{3} P_{1}$ states in He-like Ti decaying into different states. The partial strengths $\left[S_{p}\left(10^{-20} \mathrm{~cm}^{2} \mathrm{eV}\right)\right]$ were calculated from the initial strengths by multiplying by the branching ratio. The initial strengths $\left(10^{-20} \mathrm{~cm}^{2} \mathrm{eV}\right)$ are taken from Table IV.

\begin{tabular}{ccccc}
\hline \hline $\begin{array}{c}\text { Transition } \\
\rightarrow\end{array}$ & $\begin{array}{c}\text { Initial } \\
\text { strength }\end{array}$ & $A_{r}$ & $\begin{array}{c}\text { Branching } \\
\text { ratio }(\%)\end{array}$ & $S_{p}$ \\
\hline $1 s 3 p{ }^{1} P_{1} \rightarrow 1 s 2 s{ }^{1} S_{0}$ & 8.38 & $1.45(13)$ & 5.6 & 0.47 \\
$\rightarrow 1 s 2 s{ }^{3} S_{1}$ & & $6.73(11)$ & 0.3 & 0.03 \\
$\rightarrow 1 s^{2}{ }^{1} S_{0}$ & & $2.41(14)$ & 94.1 & 7.89 \\
$1 s 3 p{ }^{3} P_{1} \rightarrow 1 s^{2}{ }^{1} S_{0}$ & 2.27 & $7.85(11)$ & 2.8 & 0.06 \\
$\rightarrow 1 s 2 s{ }^{3} S_{1}$ & & $1.43(13)$ & 52.9 & 1.20 \\
$\rightarrow 1 s^{2}{ }^{1} S_{0}$ & & $1.19(13)$ & 44.3 & 1.01 \\
\hline \hline
\end{tabular}

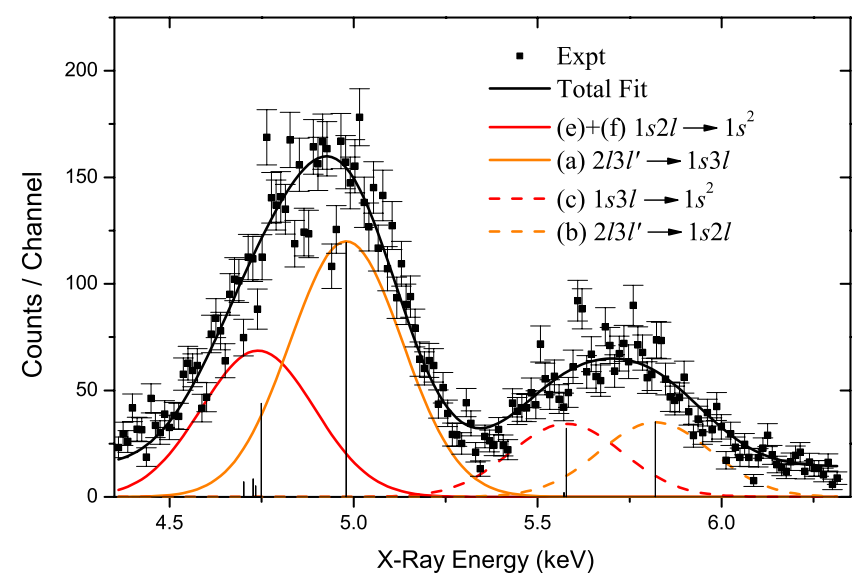

FIG. 5. (Color online) Fits to the observed x-ray spectrum from the $K L M$ DR resonance of $\mathrm{H}$-like Ti. The energies and relative intensities of the individual components comprising the four line profiles are indicated with thin vertical bars. Labels are the same as those given in Fig. 3. Red (dark gray) lines correspond to transitions to the ground state $1 s 2 l, 1 s 3 l \rightarrow 1 s^{2}$ whereas orange (light gray) lines correspond to the initial transition from a doubly excited state $2 l 3 l^{\prime} \rightarrow 1 s 3 l, 1 s 2 l$

the theoretical estimation but the ratio of these two peaks was allowed to vary. The fitting was found to be fairly sensitive to the $\mathrm{x}$-ray energy scale. Taking into account the uncertainties in the energy scale $(\approx 10 \mathrm{eV})$, the ratio of the observed secondary to the primary $\mathrm{x}$-ray intensities is found to be $0.63 \pm 0.15$. This should be compared with the theoretically estimated number $[0.795$ as in Eq. (6)] given above. This ratio can also be expressed as a fraction of the normalized total intensity and is given in Table VI. For the $K L L$ manifold the predicted ratios are in reasonable agreement with the observed values.

The $\mathrm{x}$-ray spectrum due to decay of the $K L M$ resonance of the He-like ion is assumed to consist of the four main groups of transitions labeled $a, b, c$, and $e+f$ in Fig. 3. A portion of the observed spectrum is shown in Fig. 5 along with a fit to the data. Because a large number of transitions with small energy separation are involved in the $a$ and $b$ groups, the observed $\mathrm{x}$-ray peaks were fitted with a single

TABLE VI. Calculated and measured normalized $\mathrm{x}$-ray intensities of transition groups for both the $K L L$ and the $K L M$ manifold. In both cases the $2 E 1$ transitions are excluded from the theoretical calculation. Measured ratios are derived from peak fitting using calculated partial strengths and $\mathrm{x}$-ray energies for individual transitions as described in the text.

\begin{tabular}{llcc}
\hline \hline & \multicolumn{2}{c}{ Transition } & \multicolumn{2}{c}{$\begin{array}{c}\text { Normalized ratios } \\
\text { Measured }\end{array}$} & Theoretical \\
\hline \multirow{2}{*}{$K L L$} & $2 l 2 l^{\prime} 1 s 2 l$ & $0.61 \pm 0.05$ & 0.5556 \\
& $1 s 2 l 1 s^{2}$ (excluding, 2E1) & $0.39 \pm 0.05$ & 0.4444 \\
$K L M$ & $2 l 3 l^{\prime} 1 s 3 l(a)$ & $0.47 \pm 0.12$ & 0.437 \\
& $2 l 3 l^{\prime} 1 s 2 l(b)$ & $0.13 \pm 0.05$ & 0.071 \\
& $1 s 2 l 1 s^{2}(e+f$, excluding, 2E1) & $0.27 \pm 0.07$ & 0.346 \\
& $1 s 3 l 1 s^{2}(c)$ & $0.13 \pm 0.05$ & 0.146 \\
\hline \hline
\end{tabular}


Gaussian function. The x-ray energy of these peaks was calculated by using a weighted average. Only two transitions contribute to the peak of the decay channel $c$ and accordingly two separate Gaussians were used to fit this contribution. The $e+f$ peak consists of four Gaussian functions corresponding to decay of the four individual $1 s 2 l$ states. The overall fit is then composed of eight individual Gaussian peaks but there are only four free parameters corresponding to the intensity of each of the four groups of transitions.

From fitting, the experimental $x$-ray intensity ratios among different transition channels were determined and the results converted to normalized fractions in the same way as the KLL manifold. Results are given in Table VI. For all the transition groups the theoretical normalized fractions agree reasonably well with the experimentally determined fractions. It should be noted that the calculated theoretical ratios did not include polarizations of the $\mathrm{x}$ rays as they are not known well; however, inclusion should produce only slight modifications. From the fit the measured branching ratio for decay from the $1 s 3 l$ state was also determined:

$$
I(c) / I(d)=I(c) /[I(e)+I(f)-I(b)]=0.9 \pm 0.8,
$$

which may be compared to the theoretical prediction of 0.50 . However, the large errors for each measured group, especially the smallest $2 l 3 l^{\prime} \rightarrow 1 s 3 l(b)$ manifold, combine to make the final error in this ratio rather large in this case.

\section{CONCLUSION}

In the present work, $K L L$ and $K L M$ DR into H-like titanium $\left(\mathrm{Ti}^{21+}\right)$ ions has been experimentally investigated and also theoretically analyzed. Through sequential decays of the doubly excited He-like ion formed through DR into H-like ions, two $\mathrm{x}$ rays with different energies were observed and their energy and intensity distributions were analyzed within the limited energy resolution of the detector. These sequential decays produce more than one $\mathrm{x}$ ray per DR event, resulting in significantly enhanced $\mathrm{x}$-ray yields. This aspect is quite important from the point of view of the energy loss from high-temperature plasmas. Reasonable agreement was found between the calculated and measured X-ray intensity distributions for both the $K L L$ and $K L M$ DR resonance manifolds. Increased statistics and detector resolution could allow this method to become a more sensitive test of current theory.

The DR process into $\mathrm{H}$-like ions includes a number of interesting atomic physics issues related to understanding of the decay of excited few-electron ions. Further detailed studies with heavy, highly charged ions are worthwhile and should be pursued. Future experiments could also use two detectors in an attempt to observe both $\mathrm{x}$ rays, in coincidence, from the doubly and singly excited states originated from DR processes, including cascade as well as $2 E 1$ transitions, as has already been done for $K$-shell vacancies in silver ions [44].

\section{ACKNOWLEDGMENTS}

F.J.C. and B.E.O'R. acknowledge financial support from the Royal Society, the Great Britain Sasakawa Foundation, and the British Council for travel funding which made this collaboration possible. This work was performed under the auspices of the International Cooperative Research Project (ICORP) of the Japan Science and Technology Corporation. H.T. also acknowledges Atlantic Philanthropies for financial assistance during his stay in Belfast.
[1] Y. Hahn, Rep. Prog. Phys. 60, 691 (1997).

[2] D. W. Savin and J. M. Laming, Astrophys. J. 566, 1166 (2002).

[3] K. Seon, U. Nam, and I. H. Park, J. Phys. B 36, 2679 (2003).

[4] D. E. Post, J. Nucl. Mater. 220, 143 (1995).

[5] C. Brandau et al., Phys. Rev. Lett. 91, 073202 (2003).

[6] S. Kieslich, S. Schippers, W. Shi, A. Müller, G. Gwinner, M. Schnell, A. Wolf, E. Lindroth, and M. Tokman, Phys. Rev. A 70, 042714 (2004).

[7] G. Kilgus et al., Phys. Rev. Lett. 64, 737 (1990).

[8] C. Brandau, et al. GSI scientific report, 2002, p. 91 (unpublished).

[9] C. Brandau et al., Radiat. Phys. Chem. 75, 1763 (2006).

[10] M. W. Clark, J. A. Tanis, E. M. Bernstein, N. R. Badnell, R. D. DuBois, W. G. Graham, T. J. Morgan, V. L. Plano, A. S. Schlachter, and M. P. Stockli, Phys. Rev. A 45, 7846 (1992).

[11] X. Ma, P. H. Mokler, F. Bosch, A. Gumberidze, C. Kozhuharov, D. Liesen, D. Sierpowski, Z. Stachura, T. Stöhlker, and A. Warczak, Phys. Rev. A 68, 042712 (2003).

[12] R. Ali, C. P. Bhalla, C. L. Cocke, and M. Stockli, Phys. Rev. Lett. 64, 633 (1990).

[13] R. Ali, C. P. Bhalla, C. L. Cocke, M. Schulz, and M. Stockli,
Phys. Rev. A 44, 223 (1991).

[14] D. R. DeWitt, D. Schneider, M. W. Clark, M. H. Chen, and D. Church, Phys. Rev. A 44, 7185 (1991).

[15] D. R. DeWitt, D. Schneider, M. H. Chen, M. W. Clark, J. W. McDonald, and M. B. Schneider, Phys. Rev. Lett. 68, 1694 (1992).

[16] D. R. DeWitt, D. Schneider, M. H. Chen, M. B. Schneider, D. Church, G. Weinberg, and M. Sakurai, Phys. Rev. A 47, R1597 (1993).

[17] H. Watanabe, H. Tobiyama, A. P. Kavanagh, Y. M. Li, N. Nakamura, H. A. Sakaue, F. J. Currell, and S. Ohtani, Phys. Rev. A 75, 012702 (2007).

[18] A. P. Kavanagh, H. Watanabe, Y. M. Li, H. Tobiyama, N. Nakamura, S. McMahon, C. Yamada, S. Ohtani, and F. J. Currell (unpublished).

[19] A. J. Smith, P. Beiersdorfer, K. Widmann, M. H. Chen, and J. H. Schofield, Phys. Rev. A 62, 052717 (2000).

[20] D. A. Knapp, R. E. Marrs, M. B. Schneider, M. H. Chen, M. A. Levine, and P. Lee, Phys. Rev. A 47, 2039 (1993).

[21] D. A. Knapp, P. Beiersdorfer, M. H. Chen, J. H. Scofield, and D. Schneider, Phys. Rev. Lett. 74, 54 (1995).

[22] H. Watanabe, F. J. Currell, H. Kuramoto, Y. M. Li, S. Ohtani, 
B. O'Rourke, and X. M. Tong, J. Phys. B 34, 5095 (2001).

[23] B. E. O'Rourke, H. Kuramoto, Y. M. Li, S. Ohtani, X. M. Tong, H. Watanabe, and F. J. Currell, J. Phys. B 37, 2343 (2004).

[24] Y. Zou, J. R. Crespo López-Urrutia, and J. Ullrich, Phys. Rev. A 67, 042703 (2003).

[25] X. Zhang, J. R. Crespo López-Urrutia, P. Guo, V. Mironov, X. Shi, A. J. González Martínez, H. Tawara, and J. Ullrich, J. Phys. B 37, 2277 (2004).

[26] T. Fuchs, C. Biedermann, R. Radtke, E. Behar, and R. Doron, Phys. Rev. A 58, 4518 (1998).

[27] W. D. Chen et al., Phys. Plasmas 14, 103302 (2007).

[28] H. Watanabe et al., Nucl. Instrum. Methods Phys. Res. B 235, 261 (2005).

[29] Z. Harman, V. Mäckel, A. J. González Martínez, A. N. Artemyev, J. R. Crespo López-Urrutia, U. D. Jentschura, C. H. Keitel, H. Tawara, I. I. Tupitsyn, J. Ullrich, ECAMP IX Book of Abstracts, Th3-8 (EPS, 2007), p. 663.

[30] A. J. González Martínez et al., Phys. Rev. A 73, 052710 (2006).

[31] Z. Harman, I. I. Tupitsyn, A. N. Artemyev, U. D. Jentschura,
C. H. Keitel, J. R. Crespo López-Urrutia, A. J. González Martínez, H. Tawara, and J. Ullrich, Phys. Rev. A 73, 052711 (2006).

[32] F. J. Currell et al., J. Phys. Soc. Jpn. 65, 3186 (1996).

[33] F. J. Currell et al., Phys. Scr. T 73, 371 (1997).

[34] K. LaGattuta and Y. Hahn, J. Phys. B 15, 2101 (1982).

[35] M. H. Chen and J. H. Scofield, Phys. Rev. A 52, 2057 (1995).

[36] G. W. F. Drake, Phys. Rev. A 34, 2871 (1986).

[37] C. D. Lin, W. R. Johnson, and A. Dalgarno, Phys. Rev. A 15, 154 (1977).

[38] H. Gould, R. Marrus, and R. W. Schmieder, Phys. Rev. Lett. 31, 504 (1973).

[39] G. W. F. Drake, Phys. Rev. A 19, 1387 (1979).

[40] U. Fano and J. H. Macek, Rev. Mod. Phys. 45, 553 (1973).

[41] P. Beiersdorfer, National Institute for Fusion Science, Toki, Japan, Report No. PROC-37, 1998 (unpublished), p. 67.

[42] P. Beiersdorfer et al., Phys. Rev. A 53, 3974 (1996).

[43] I. I. Sobelman, Atomic Spectra and Radiative Transitions (Springer, Berlin, 1992).

[44] P. H. Mokler, H. W. Schäffer, and R. W. Dunford, Phys. Rev. A 70, 032504 (2004). 